\title{
TITLE:
}

\section{Indium nitride thin films prepared by radio-frequency reactive sputtering}

$\operatorname{AUTHOR}(\mathrm{S})$ :

MARUYAMA, T; MORISHITA, T

\section{CITATION:}

MARUYAMA, T ... [et al]. Indium nitride thin films prepared by radiofrequency reactive sputtering. JOURNAL OF APPLIED PHYSICS 1994, 76(10): 5809-5812

\section{ISSUE DATE:}

1994-11-15

URL:

http://hdl.handle.net/2433/43535

\section{RIGHT:}

Copyright 1994 American Institute of Physics. This article may be downloaded for personal use only. Any other use requires prior permission of the author and the American Institute of Physics. 


\title{
Indium nitride thin films prepared by radio-frequency reactive sputtering
}

\author{
Toshiro Maruyama and Tomonori Morishita \\ Department of Chemical Engineering, Faculty of Engineering, Kyoto University, Yoshida-Honmachi, \\ Sakyo-ku, Kyoto 606, Japan
}

(Received 9 May 1994; accepted for publication 30 July 1994)

\begin{abstract}
Indium nitride thin films were obtained by the reactive sputtering method. The metallic indium target was sputtered by nitrogen gas with if magnetron sputtering equipment. The stoichiometric indium nitride film showed high Hall mobility $\left(363 \mathrm{~cm}^{2}(\mathrm{~V} \mathrm{~s})^{-1}\right)$ and low carrier concentration $\left(5.98 \times 10^{18} \mathrm{~cm}^{-3}\right)$. To suppress the nitrogen vacancies, the reevaporation of atomic nitrogen from the substrate must be suppressed by lowering the substrate temperature and the resputtering of atomic nitrogen must be minimized by adjusting the total pressure close to the upper limit of the effective pressure for deposition.
\end{abstract}

\section{INTRODUCTION}

Indium nitride ( $\mathrm{InN}$ ) is a direct-band-gap III-V compound semiconductor. The InN thin film has a vast potential for device applications, because of its direct band gap (1.89 $\mathrm{eV}$ ) and high mobility. The InN film has been prepared by reactive sputtering methods ${ }^{1-3}$ and reactive evaporation. ${ }^{4}$ The prepared film sometimes incorporated a vast number of nitrogen vacancies as donor centers, showing lower mobilities of the order of $1 \mathrm{~cm}^{2}(\mathrm{~V} \mathrm{~s})^{-1}$ and carrier concentrations above $10^{20} \mathrm{~cm}^{-3}$. Hovel and Cuomo ${ }^{1}$ used a reactive sputtering method to prepare high-quality films with a Hall mobility of $250 \pm 50 \mathrm{~cm}^{2}(\mathrm{~V} \mathrm{~s})^{-1}$ and carrier concentration of $(5-8) \times 10^{18} \mathrm{~cm}^{-3}$. Using rf sputtering of a indium target in a reactive nitrogen plasma, Foley and Tansley ${ }^{3}$ reported a Hall mobility of $5000 \mathrm{~cm}^{2}(\mathrm{~V} \mathrm{~s})^{-1}$ and a carrier concentration of $3 \times 10^{16} \mathrm{~cm}^{-3}$ (at $150 \mathrm{~K}$ ); however, the deposition conditions have not been systematically investigated to obtain films of these high Hall mobilities and low carrier concentrations.

Our objective is to prepare InN thin films of high Hall mobilities and low carrier concentrations by using rf reactive sputtering of a indium target. The sputtering parameters used were substrate temperature, if power, and total pressure. The deposition condition is discussed on the basis of the measured composition and deposition rate of the film.

\section{EXPERIMENTS}

In preparing InN films, if (13.5 $\mathrm{MHz})$ magnetron sputtering equipment (Osaka Vacuum Co., Ltd.) was used with a $99.99 \%$ pure indium target $10 \mathrm{~cm}$ in diameter and $1 \mathrm{~mm}$ thick. The of power was $10-500 \mathrm{~W}$, the sputtering gas was a 99.999\% pure nitrogen, and its flow rate ranged $2-200 \mathrm{sccm}$. The separation distance between the substrate and the targel was $48 \mathrm{~mm}$. The chamber vacuum just before growth was less than $3.0 \times 10^{-6}$ Torr. The substrate temperature ranged $20-470^{\circ} \mathrm{C}$. It was measured using a Chromel-Alumel thermocouple attached to the front of the substrate holder. The total sputtering pressure ranged $0.3-25$ mTorr. A $76 \times 26$ $\mathrm{mm}^{2}$ borosilicate glass plate was used as the substrate.

The composition of the film was measured by $x$-ray photoelectron spectroscopy, and was expressed as the atomic ratio of nitrogen to indium. The measured atomic ratio was calibrated by the measurement of stoichiometric indium nitride powder (purity of $99.9 \%$ ). The crystallinity of the film was analyzed by $\mathrm{x}$-ray-diffraction with $\mathrm{Cu} K \alpha$ radiation. The electric resistivity of the film was measured by the van der Pauw method. The carrier concentration and Hall mobility were measured by using the Hall effect.

\section{RESULTS AND DISCUSSION}

The films were transparent and colored dark red. All samples were $n$-type semiconductor of high conductivity. Figure 1 shows a typical example of the $x$-ray-diffraction pattern of the film on a borosilicate glass. The precise (002) and (004) peaks indicate that the film is composed of crystallites with a hexagonal wurtzite structure. A large peak of (002) indicates that the film is highly $c$-axis oriented. All samples showed the similar $x$-ray-diffraction patterns, indicating the highly $c$-axis-oriented hexagonal wurtzite structure.

Figure 2 shows the resistivity, Hall mobility, and carrier concentration of the film as a function of substrate temperature. The results were obtained at fixed values of the rf power $(200 \mathrm{~W})$ and the total pressure $(1.7 \mathrm{~m}$ Torr $)$. All over the temperature range the Hall mobility is lower than 12 $\mathrm{cm}^{2}(\mathrm{~V} \mathrm{~s})^{-1}$ and the carrier concentration is higher than $10^{20}$ $\mathrm{cm}^{-3}$. The lowest substrate temperature can be selected as

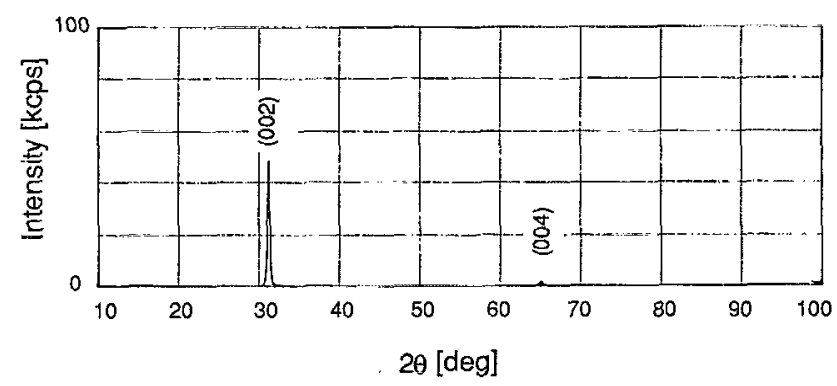

FIG. 1. X-ray-diffraction pattern of 260 -nm-thick $\mathrm{InN}$ film prepared at a total pressure of $1.7 \mathrm{mTorr}$, substrate temperature of $60^{\circ} \mathrm{C}$, and if power of $40 \mathrm{~W}$. 


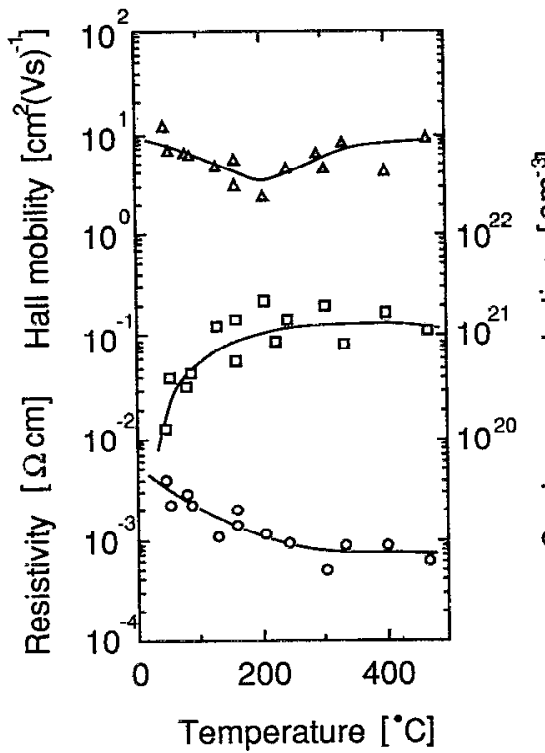

FIG. 2. Electric resistivity, Hall mobility, and carrier concentration as a function of substrate temperature.

the optimum temperature, because of its lowest carrier concentration and highest Hall mobility. Figure 3 shows the atomic ratio of nitrogen to indium as a function of substrate temperature. The lowest carrier concentration at the lowest substrate temperature is characterized by the highest value of the atomic ratio, although the ratio is still lower than the stoichiometric ratio, $\mathrm{N} / \mathrm{In}=1$. The substrate temperature of about $60^{\circ} \mathrm{C}$ was selected as the lowest temperature, which was controllable in the experiments over wide ranges of deposition variables under the effect of plasma heating. In the following discussion, the results are based on the films prepared at a substrate temperature of about $60^{\circ} \mathrm{C}$.

Figure 4 shows the resistivity, Hall mobility, and carrier concentration of the film as a function of if power. The results were obtained at fixed values of the total pressure ( 1.7 mTorr) and substrate temperature (about $60^{\circ} \mathrm{C}$ ). All over the rf power range, the Hall mobility is lower than 33

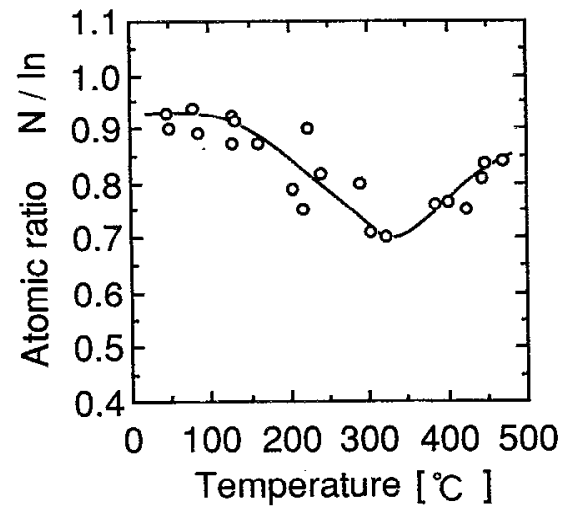

FIG. 3. Atomic ratio of nitrogen to indium as a function of substrate temperature.

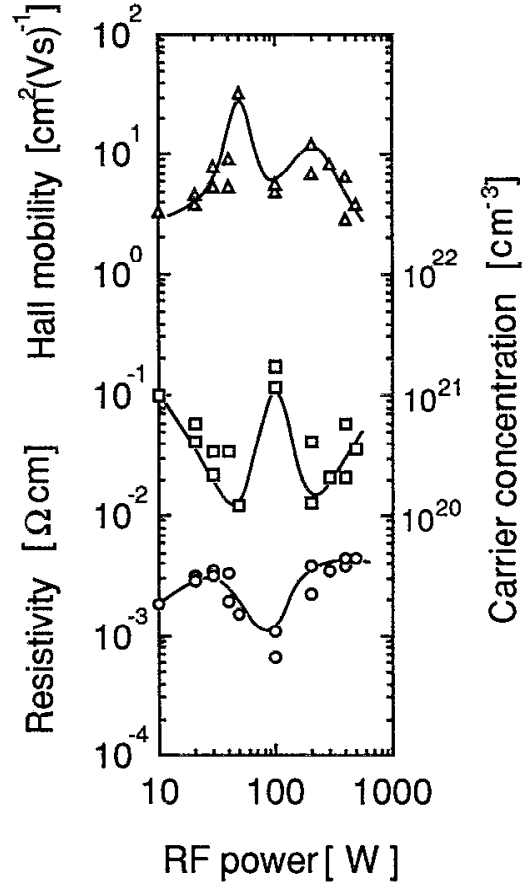

FIG. 4. Electric resistivity, Hall mobility, and carrier concentration as a function of if power.

$\mathrm{cm}^{2}(\mathrm{~V} \mathrm{~s})^{-1}$ and the carrier concentration is higher than $10^{20}$ $\mathrm{cm}^{-3}$. The rf power of either 50 or $200 \mathrm{~W}$ can be selected as the optimum if power, because of the minimum values of the carrier concentration and the maximum values of the Hall mobility. Figure 5 shows the atomic ratio $\mathrm{N} / \mathrm{In}$ as a function of rf power. The minimum value of the carrier concentration at one of the optimum if powers, $200 \mathrm{~W}$, is characterized by the highest atomic ratio, although the ratio is still less than the stoichiometric ratio, $\mathrm{N} / \mathrm{In}=1$. Therefore, $200 \mathrm{~W}$ is selected as the optimum if power.

Figure 6 shows the resistivity, Hall mobility, and carrier concentration of the film as a function of total pressure. The results were obtained at the fixed values of if power $(200 \mathrm{~W})$ and substrate temperature (about $60^{\circ} \mathrm{C}$ ). At a pressure of 7

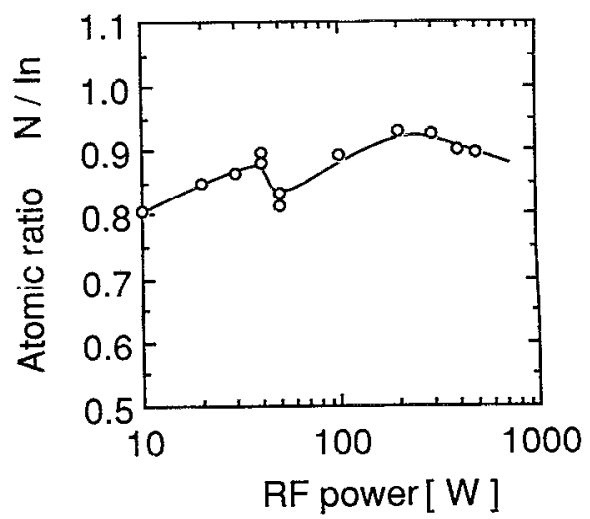

FIG. 5. Atomic ratio of nitrogen to indium as a function of if power. 


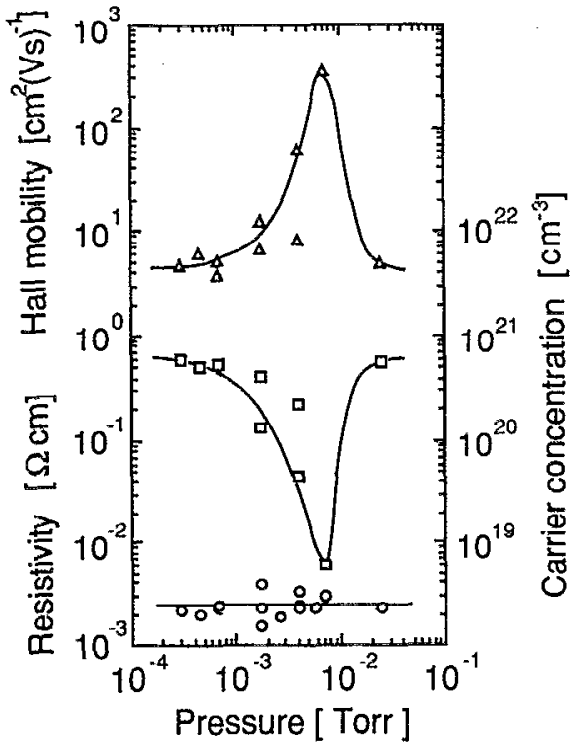

FIG. 6. Electric resistivity, Hall mobility, and carrier concentration as a function of total pressure.

mTorr, the Hall mobility takes the maximum value, 363 $\mathrm{cm}^{2}(\mathrm{~V} \mathrm{~s})^{-1}$, and the carrier concentration takes the minimum value, $5.98 \times 10^{18} \mathrm{~cm}^{-3}$. These values are close to those reported by Hovel and Cuomo. ${ }^{1}$ Thus, the total pressure of 7 mTorr, If power of $200 \mathrm{~W}$, and substrate temperature of about $60^{\circ} \mathrm{C}$ are the optimum conditions for obtaining the lowest carrier concentration and highest Hall mobility. Figure 7 shows the atomic ratio N/In as a function of total pressure. The minimum value of the carrier concentration at the optimum total pressure, 7 mTorr, is characterized by the atomic ratio 0.983 , which is very close to the stoichiometric ratio $\mathrm{N} / \mathrm{In}=1$. Figure 8 shows the deposition rate of the film as a function of total pressure. The deposition rate, which is high and nearly constant in the low-pressure region, decreases dramatically above about 7 mTorr. Thus, the optimum total pressure, $7 \mathrm{mTorr}$, is close to the upper limit of the effective total pressure for deposition.

On the basis of the above-mentioned optimum conditions for deposition, the deposition mechanisms of the sto-

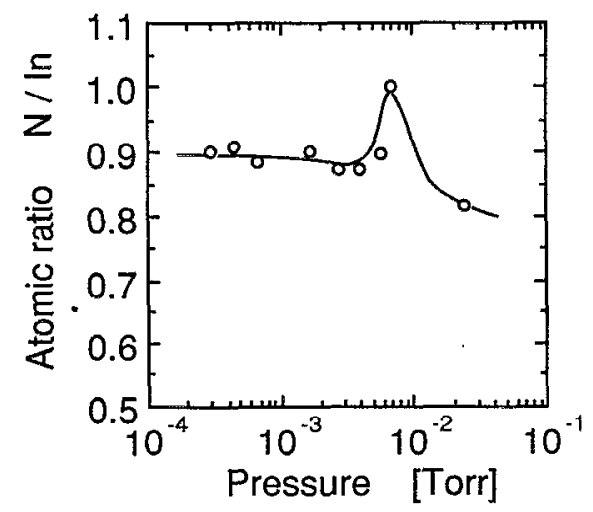

FIG. 7. Atomic ratio of nitrogen to indium as a function of total pressure.

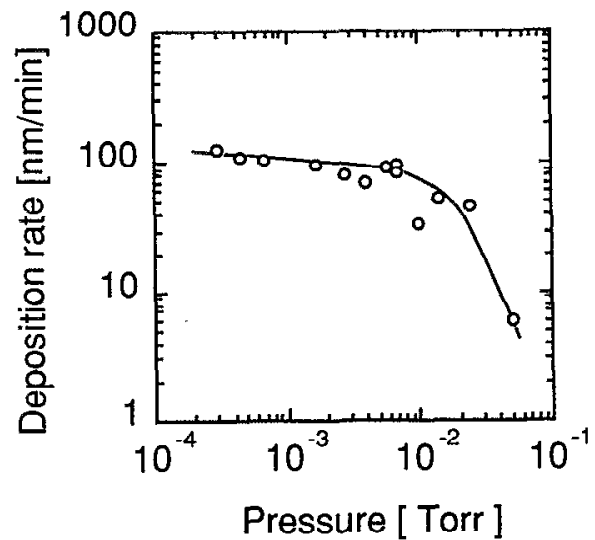

FIG. 8. Deposition rate of $\mathrm{InN}$ film as a function of total pressure.

ichiometric InN film, which shows high Hall mobility and low carrier concentration, are qualitatively described as follows.

According to Natarajan and co-workers, ${ }^{2}$ indium chemisorbs not molecular nitrogen but atomic nitrogen, and the reactive atomic nitrogen is supplied by the following reaction:

$$
\mathrm{N}_{2}^{+}+\mathrm{N}_{2} \rightarrow \mathrm{N}_{2}+\mathrm{N}^{+}+\mathrm{N} \text {. }
$$

Visual observations showed that the surface of the target was always colored black after each run; thus, a nitrided layer was formed on the target. In addition, the films were exclusively $c$-axis oriented and polycrystalline (see Fig. 1). According to Foley and Tansley, ${ }^{3}$ this fact indicates that the partial pressure of atomic nitrogen is high enough in the of discharge region close to the target, and consequently that the layer on the target is fully nitrided. Even though the sputtered layer is a stoichiometric InN, however, the deposited film is sometimes nitrogen deficient and is not always stoichiometric. This is attributable to the surface adatom reemissions: i.e., the reevaporation and resputtering of atomic nitrogen from the substrate. In fact, the films are nitrogen deficient at higher substrate temperatures as shown in Fig. 3. Thus, one of the optimum conditions, the lowest substrate temperature, is effective in reducing the reevaporation of the atomic nitrogen. In the meantime the substrates in sputtering systems are nearly always subject to a self-induced negative vias, resulting in ion bombardments to the substrate. This, in turn, leads to the resputtering of the atomic nitrogen from the substrate. $^{5}$ The other optimum condition, the higher total pressure, is effective in reducing the resputtering, because the self-induced negative vias decrease with increasing total pressure. ${ }^{5}$ When the total pressure is excessively high, however, the deposition rate decreases (see Fig. 8), because the ionic nitrogen $\mathrm{N}_{2}^{+}$, which reaches the target, decreases by the collision with molecular nitrogen $\mathrm{N}_{2}$, as shown in Eq. (1). That is, the optimum total pressure, 7 mTorr, is close to the upper limit of the effective pressure for deposition, i.e., the highest pressure just before the sputtering rate of indium diminished dramatically. 


\section{CONCLUSIONS}

The indium nitride thin films of high Hall mobility and low carrier concentrations were obtained by the reactive sputtering method. To suppress the nitrogen vacancies, the reevaporation of atomic nitrogen from the substrate must be suppressed by lowering the substrate temperature and the resputtering of atomic nitrogen must be minimized by adjusting the total pressure close to the upper limit of the effective pressure for deposition.

\section{ACKNOWLEDGMENTS}

This work was supported by the Ookura Foundation. The authors thank K. Nakanishi and T. Yamaoka of Nippon Sheet Glass Co., Ltd. for the Hall coefficient measurements.

${ }^{1}$ H. J. Hovel and J. J. Cuomo, Appl. Phys. Lett. 20, 71 (1972).

${ }^{2}$ B. R. Natarajan, A. H. Eltoukhy, and J. E. Greene, Thin Solid Films 69, 201 (1980).

${ }^{3}$ C. P. Foley and T. L. Tansley, Appl. Surf. Sci. 22/23, 663 (1985).

${ }^{4}$ J. W. Tanier and K. Rosc, J. Electron. Mater. 3, 821 (1974).

${ }^{5}$ J. L. Vossen and J. J. Cuomo, Thin Film Processes (Academic, New York, 1978), p. 50. 